\title{
An Empirical Study on the Influence of Dormitory on College Students' Social Network: Based on Social Network Analysis
}

\author{
Wu Ling, Liao Kangli* \\ Management College, Jiujiang University, Jiujiang City, China \\ Email address: \\ 29461317@qq.com (Wu Ling), liaokangli@126.com (Liao Kangli) \\ ${ }^{*}$ Corresponding author
}

\section{To cite this article:}

Wu Ling, Liao Kangli. An Empirical Study on the Influence of Dormitory on College Students' Social Network: Based on Social Network Analysis. Teacher Education and Curriculum Studies. Vol. 6, No. 2, 2021, pp. 66-72. doi: 10.11648/j.tecs.20210602.14

Received: June 7, 2021; Accepted: June 23, 2021; Published: June 29, 2021

\begin{abstract}
Student dormitory is an important place for students' daily life and study, and also the forefront of Ideological and political education. This paper takes the students of class a1418 and class A1332 in Jiujiang University as the research objects, and uses the social network analysis method to carry out the tracking experimental research on students' daily social interaction. It is found that the dormitory is the key variable of the clustering effect of students' network community. This study shows that dormitory plays a key role in the formation and change of College Students' social network. Dormitory is the most important factor in the clustering of social network. As the most important place for college students' Extracurricular behavior, dormitory's influence is not limited to the life level, but further penetrated into the class, which is the most important basic organization of college learning, This finding provides an empirical basis for us to further integrate the boundaries of dormitory and class, dormitory and classroom, study and life, education and teaching, management and service, ideological education and professional education. It is suggested that in the daily management and teaching organization of college students, full attention should be paid to the role of the dormitory, and the dormitory should be effectively combined with the classroom, so as to form a college career mode of "learning life, learning life".
\end{abstract}

Keywords: Dormitory, Social Network, Social Network Analysis

\section{Introduction}

Dormitories and apartments are important places for students' daily life and study, as well as important positions for ideological and political education and quality education. In 2004, the Ministry of Education issued the notice of the Ministry of education on strengthening the management of College Students' accommodation [1]. In 2005, the Ministry of Education issued the notice of the general office of the Ministry of education on Further Strengthening the management of College Students' accommodation [2], In 2007, the Ministry of Education issued the "Notice of the general office of the Ministry of education on further improving the management of College Students' accommodation" of the Department of educational thought and Administration). The above "notice" clearly requires that the management of students' accommodation should be standardized, and students should be arranged to live in dormitories and apartments according to class, so as to create a good environment and atmosphere for students' growth and development. So far, the vast majority of colleges and universities have formed a pattern of "centralized residence of departments by buildings, grades by floors, and classes by dormitories". Dormitory members are stable and have the same or similar learning contents with dormitory members, which is convenient for learning cooperation. With the reform and innovation of higher education, the role of student dormitories in the growth of college students is becoming increasingly prominent. They play an increasingly important role in serving students' study and life, and promoting the reform and development of colleges and universities.

From the current academic literature on College Students' dormitory, it is generally believed that dormitory is the most direct place for college students to participate in interpersonal communication [3]. Dormitory interpersonal relationship is an 
important part of College Students' interpersonal relationship, and it is also a small yardstick to measure college students' mental health level, interpersonal communication ability and social behavior [4]. Relevant empirical studies show that harmonious dormitory relationship is conducive to improving the quality of university career. Yang Shaoqing and Zhang Jingxia (2012) found that the subjective well-being experience of college students with harmonious dormitory interpersonal relationship is higher [5]. Zhang Dandan et al. (2012) also found that there is a close relationship between College Students' dormitory relationship and learning attitude and learning efficiency [6], Pan Wei and Gao Xuemei (2017) found that interpersonal relationship and learning adaptability have a significant impact on College Students' academic achievement, and learning adaptability plays a certain mediating role between interpersonal relationship and academic achievement [7]. Huang Yanfei and Zhang Tong (2021) discussed the practice and path of Ideological and political work into students' dormitories in Renmin University of China. Lei ting (2021) discussed the related concepts and core significance of dormitory culture based on the perspective of three complete education [9]. Liu Juan (2021) analyzed the path to correctly deal with the contradictions in students' dormitories by means of case study [10]. Jin Zhuan and Liu Changhua (2021) discussed the educational functions and methods of college dormitory management under the new situation [11]. Song Yuan (2020) takes Chengde Petroleum College as an example to discuss the exploration and practice of the guiding management mode of College Students' dormitory under the concept of establishing morality and cultivating talents [12]. Liu Qiming (2020) analyzed the construction of community management mode of student dormitory [13]. Based on the perspective of social psychology, Wang Shanshan and Li Fang (2020) discussed the interpersonal relationship of students' dormitory in Higher Vocational Colleges [14]. Huang Shaoxia (2020) has analyzed the problems in the construction of college dormitory management team [15].

This paper is based on the perspective of natural experiments, using social network analysis method to test whether college students' social network will change significantly with the change of dormitory environment. As a phased achievement of the key project of Jiangxi Province Education Science "13th five year plan" in 2019 "the mechanism and realization path of" learning dormitory "to promote the multi integration of university education", this paper aims to further analyze the influence of dormitory on students' social network and reveal its internal mechanism based on the existing relevant research and through natural experiments.

\section{Experimental Design}

\subsection{Basic Information of Research Objects}

This study selects class a1418 and class A1332 of Accounting School of Jiujiang University as the research objects. Among them, class a1418 is the excellent accounting class of the accounting college. The students of this class entered the school in the autumn of 2014 and passed the selection examination of the accounting college in June 2015. 30 students were selected from each class of accounting major in the accounting college to form a separate class. The author of this paper began to serve as the head teacher of this class in July 2015. At that time, 30 students of this class were distributed in 28 student dormitories, For example, it is difficult for the head teacher to organize activities to visit students in the dormitory. On the one hand, it is difficult to visit students in a short time due to the large number of student dormitories. On the other hand, it may cause inconvenience to other students because the class is mixed with other students; At the same time, due to the scattered residence in the dormitory and the lack of close contact among students, once some students lose contact, they can't effectively contact other students in the dormitory. If some students don't receive the notice in time, they can't get the reminder from the students; In addition, because the students in the excellent class are all excellent students selected from each class, there may be a gap between the students in the new class and those in the original dormitory. Based on this, the head teacher made an application to the accounting school in June 2016, gathered the dormitories of the outstanding class students, and studied and discussed by the Institute, and thought that the advantages of the excellent class concentrated accommodation were greater than the disadvantages. Therefore, the dormitory of the class was adjusted uniformly, and 30 dormitories of the dormitory were adjusted to 5 dormitories (12 boys were adjusted to two 6 people. 18 female students were adjusted to three six rooms. Class A1332 is an ordinary class of Auditing major in accounting college. There are 40 students in this class, which are distributed in 10 dormitories with relatively concentrated accommodation.

\subsection{Experimental Design}

This study focuses on the role of university dormitories in students' social networks, so we mainly use the social network analysis method to investigate the status of students' social networks by means of questionnaire survey, so that students can write the names of their closest classmates. The questionnaire of class a1418 was conducted twice, the first time was in March 2016, and the class was established in July 2015, that is, the first questionnaire was conducted more than half a year after the class was established; The second questionnaire was conducted in March 2017, that is, more than half a year after the adjustment of class dormitory. The questionnaire survey of class A1332 was conducted in March 2016 during the semester when the author was teaching auditing.

\section{Data Collection and Processing Methods}

\subsection{Data Collection Methods}

After knowing the dormitory number of the students and 
the names of the most closely related students through the questionnaire, the Boolean relation matrix of "classmate dormitory" and "classmate classmate" can be established, that is, in the "classmate dormitory" relation matrix, if classmate A1 lives in dormitory M1, the relation between A1 and M1 is 1 , otherwise it is 0 ; In the "classmate classmate" relationship matrix, if classmate A1 chooses classmate A2 as the closest person, the relationship between $\mathrm{A} 1$ and $\mathrm{A} 2$ is 1 , otherwise it is 0 , and so on.

\subsection{Establishment of Class Social Network Diagram and Analysis Method}

By using the social network analysis software ora, the data can be imported into the software, and the class social network diagram can be established. On this basis, the cluster fitting analysis can be carried out.

\section{Experimental Process and Results}

The social network of class A1418 formed in March 2016 is shown in Figure 1

The social network of A1332 class formed in March 2016 is shown in Figure 2.

Figure 1 and Figure 2 intuitively can not distinguish the obvious differences, and further cluster fitting analysis is carried out. Due to the lack of obvious clustering characteristics in Figure 1, it is impossible to form a clustering analysis diagram, but figure 2 shows obvious clustering characteristics after clustering fitting, as shown in Figure 3.

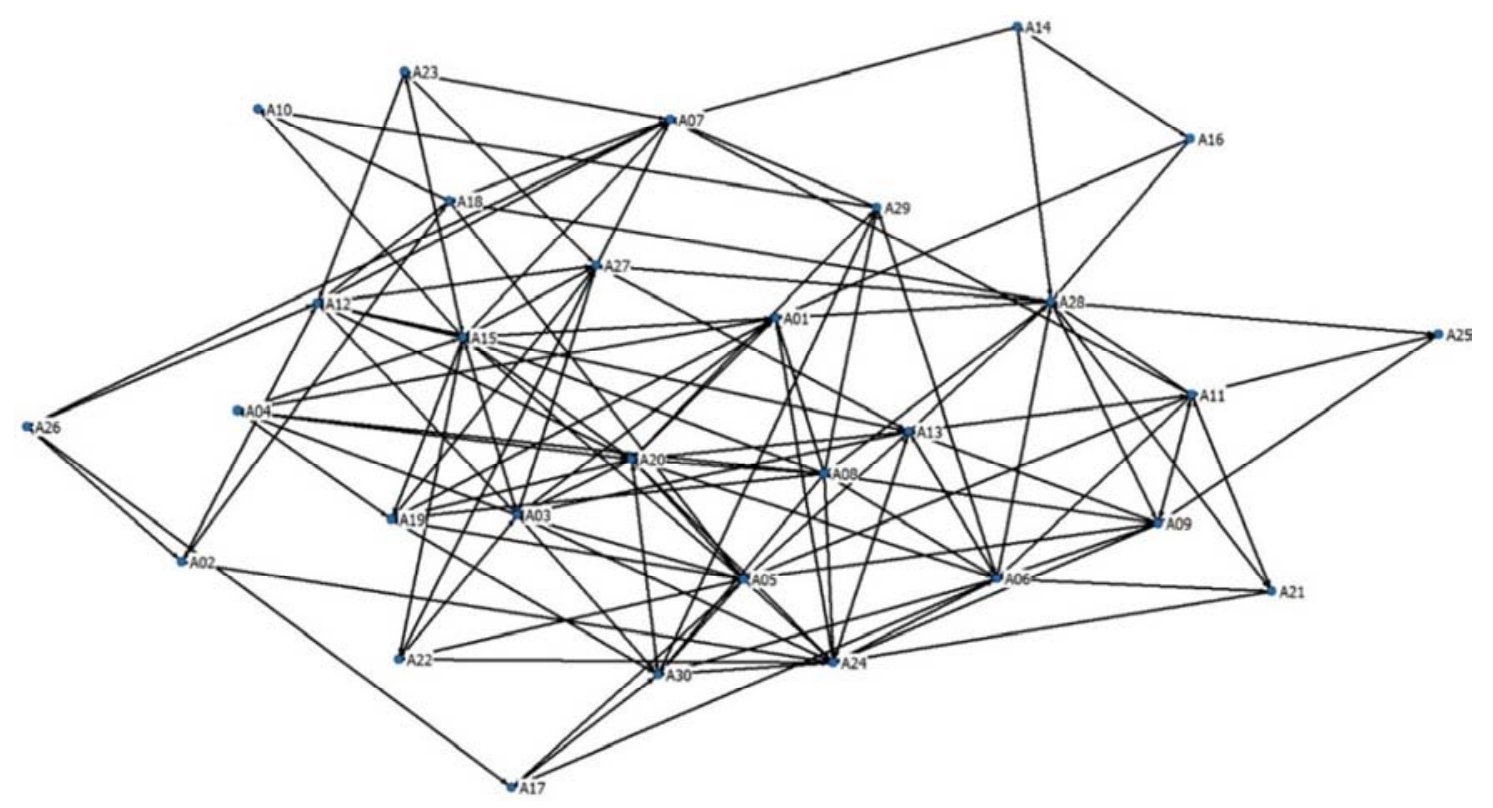

Figure 1. Social network chart of class A1418 in March 2016.

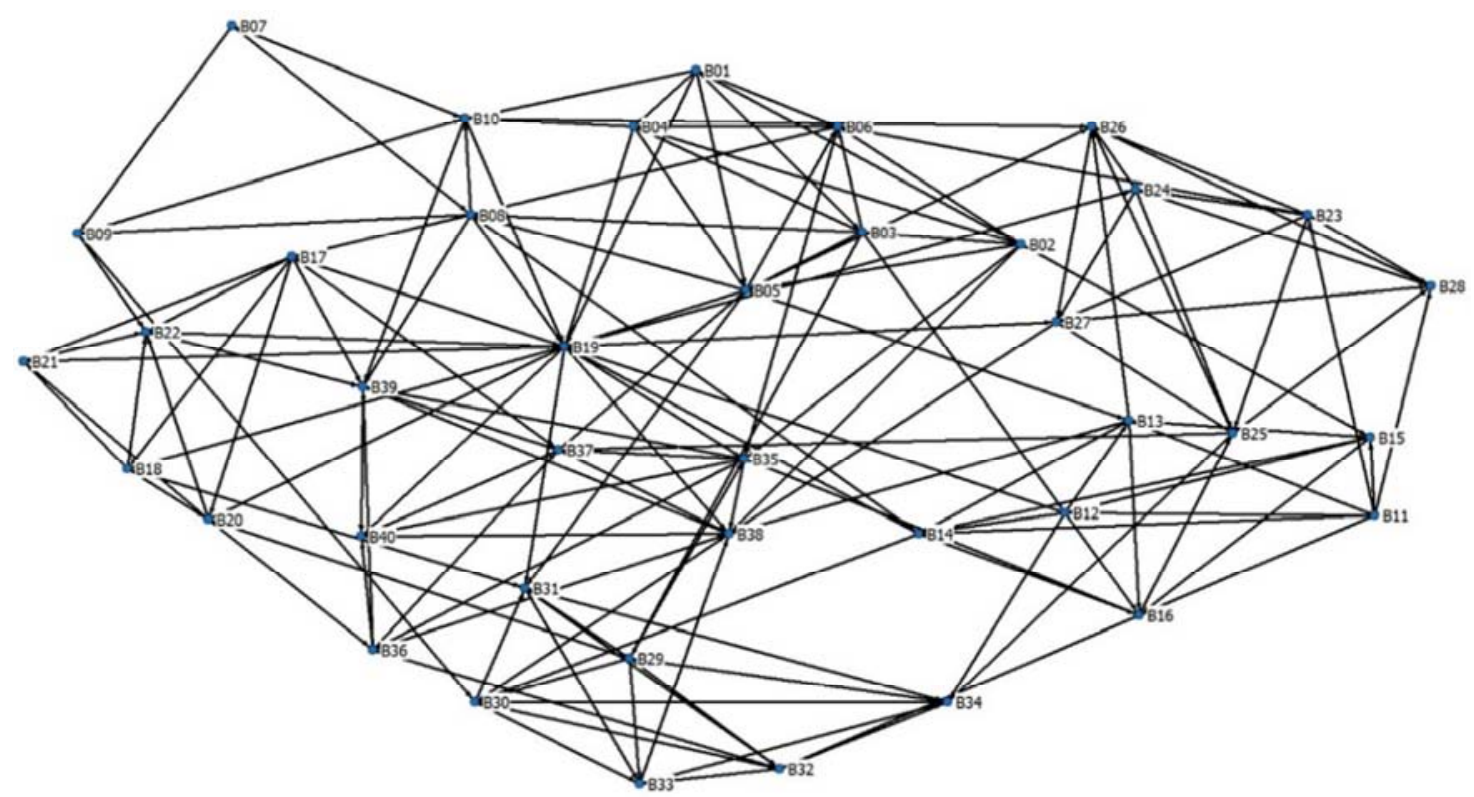

Figure 2. Social network chart of class A1332 in March 2016. 


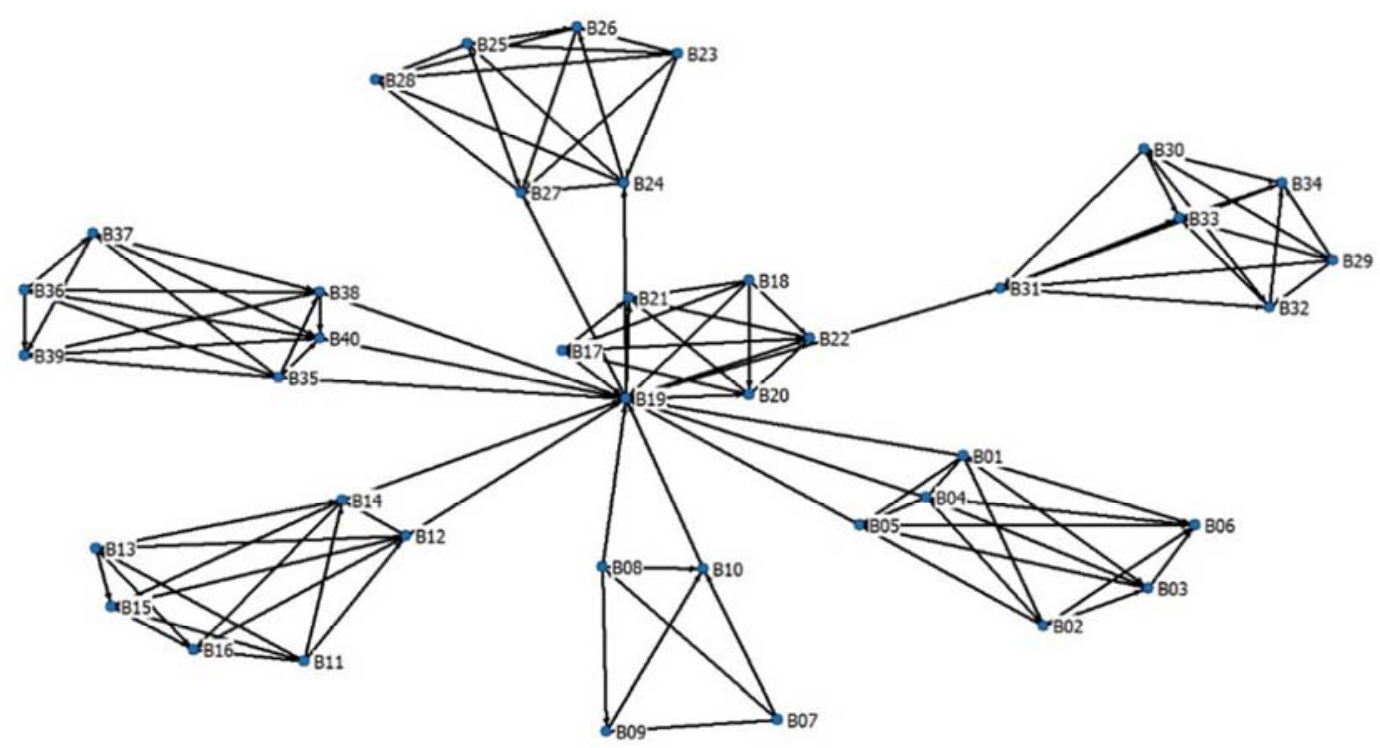

Figure 3. Cluster fitting diagram of class A1332.

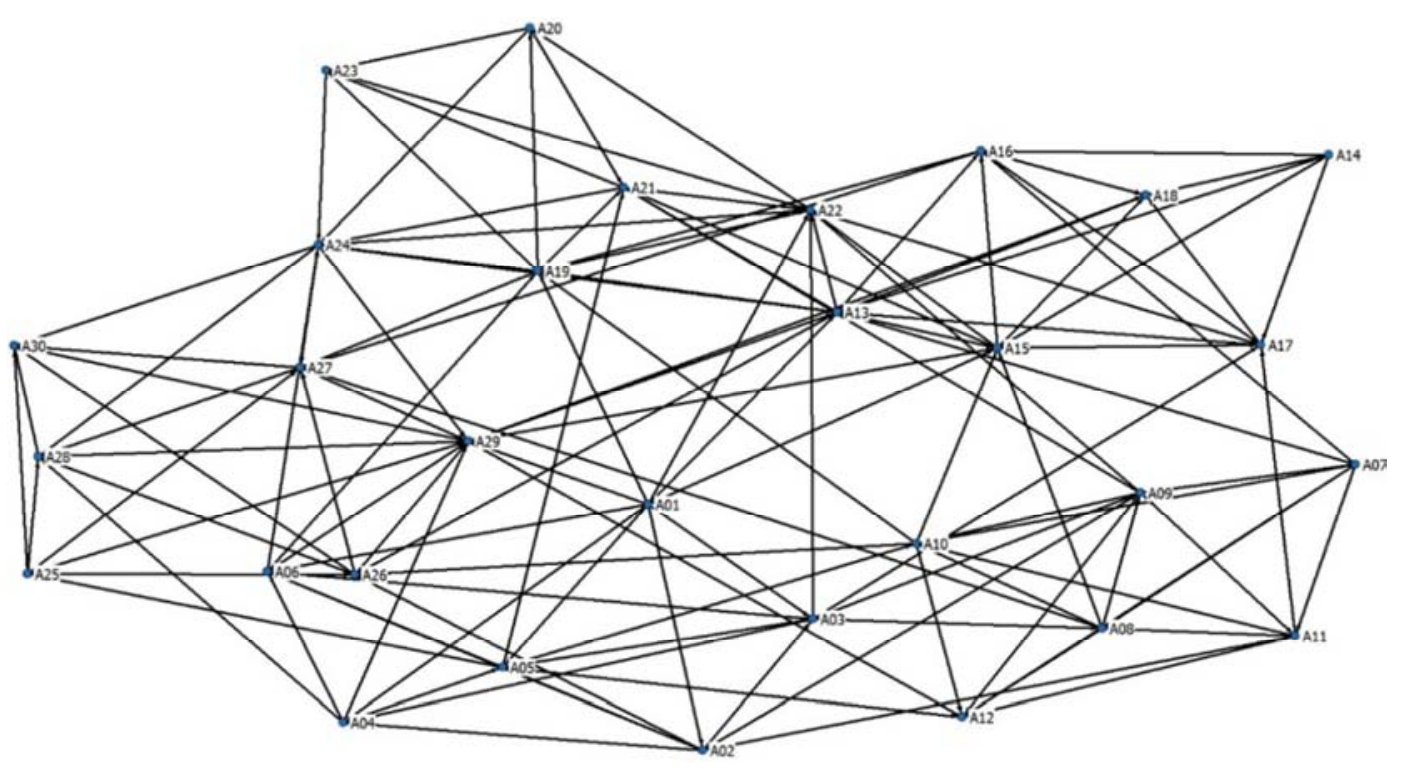

Figure 4. Social network map of class a1418 in March 2017.

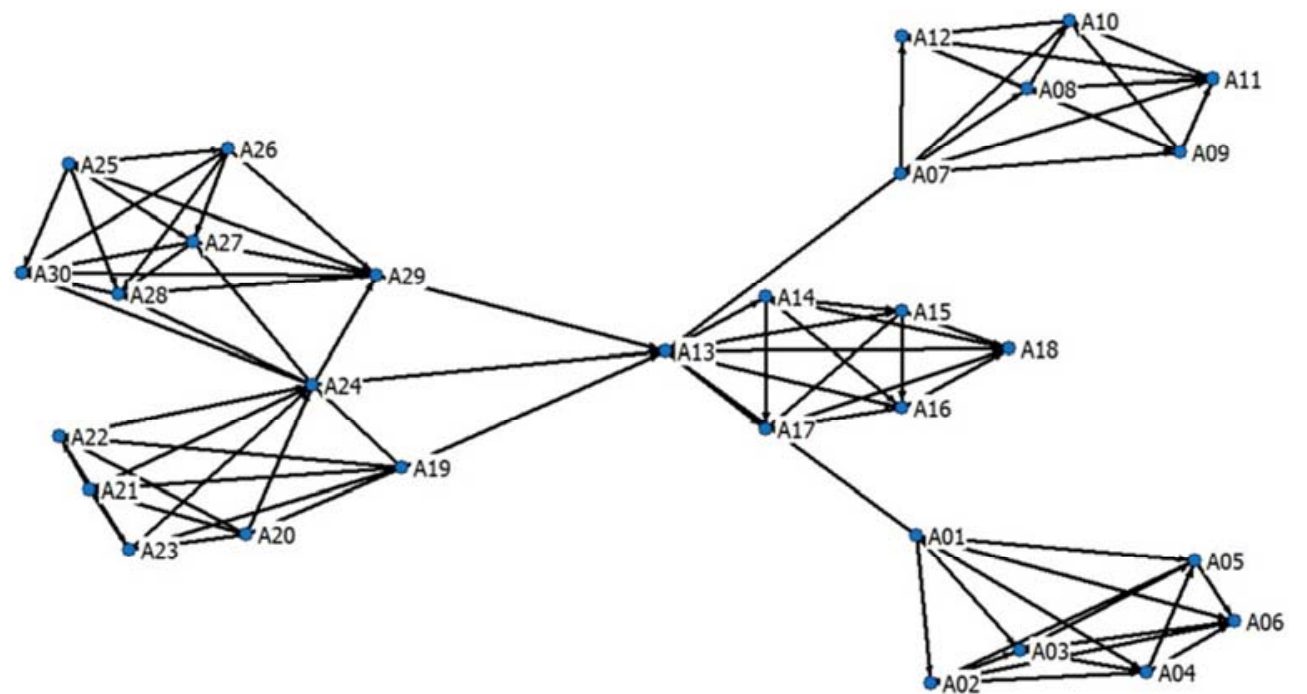

Figure 5. Cluster fitting diagram of class A1418. 


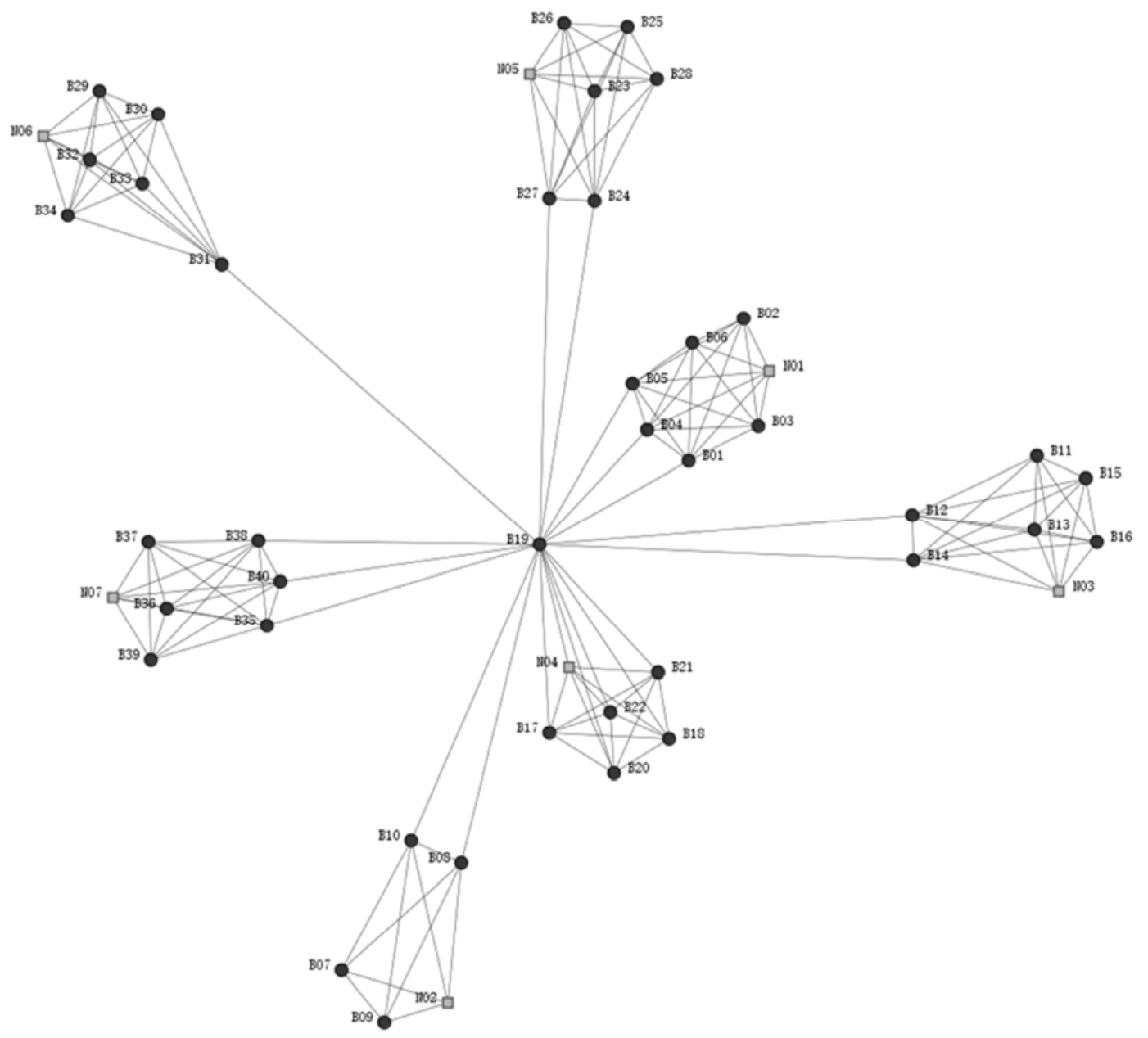

Figure 6. Class community clustering after introducing dormitory variables.

In March 2017, a questionnaire survey was conducted on class a1418, and the class community map is shown in Figure 4.

Through cluster fitting analysis of Figure 4, it is found that figure 4 has obvious clustering characteristics, as shown in Figure 5.

It can be found that dormitory is the main variable in the cluster fitting of Figure 3 and figure 5. The green square node in Figure 6 represents the dormitory. From this figure, we can see that each cluster corresponds to a dormitory.

Through further analysis, it can be found that there are other variables that affect the social relationship between class members. Through research and analysis, it can be roughly divided into the following aspects: (1) age factors, students born in the same year, especially those with close birthdays, are easy to get together (2) Students with similar family growth environment and experience are easier to get together (3) Regional factors, students with the same region and similar customs are easy to get together (4) Interest factors, students with the same interest are easy to get together, and so on. Of course, there are some other interesting answers. For example, we also find that students with adjacent student numbers are easy to get together because they often sit in the front and back of the exam; Students with similar or obvious differences are easy to get together; Students with similar or obvious facial values are easy to get together, and so on.

\section{Analysis and Conclusion}

At the end of the 19th century, Cornell University of America had a famous frog experiment, the results of which were called "frog cooking effect", and "frog cooking effect" told us that rapid environmental changes often mobilize the reaction mechanism of the body, and the most dangerous environment is slow change, The dormitory is a relatively stable learning and living environment for students during the University. In the practice of student education and management, it is often found that the "good nest" or "poor nest" phenomenon in which dormitory is the unit can be found. Therefore, the dormitory effect which gets along with each other during the University has a subtle influence on the growth of students, The influence of this kind of subtlety is mainly reflected in the following aspects: (1) dormitory is the best place for students to exchange information. Dormitory is the most convenient place for college students to exchange and evaluate information. Students can speak freely in the dormitory. Many students' dormitories communicate in a "sleeping conference" or similar form. On the one hand, this kind of communication without scruples among students is conducive to the opening up of vision, enlightening thinking 
and active thinking of dormitory members (2) Dormitory is the highland of the formation of moral and cultural quality of college students. The civilized degree and style of the small environment in the dormitory not only reflect the spirit of the college students themselves, but also have an impact on the students' physical and mental. Healthy up and colorful dormitory culture can make dormitory members get along harmoniously, form a good mood and happy emotion, and sublimate moral sentiment. Therefore, it is easy to appear "a good nest" phenomenon in a harmonious upward dormitory environment, but some students are addicted to video and video $\mathrm{CD}$ and computer games, and enjoy themselves in the dormitory; Some dormitories often talk about some vulgar topics, vent negative emotions, make dormitory crooked and evil atmosphere rise, low-level interest spread. This causes some college students to speak in public places rather elegant, but back to the dormitory but have no scruples, between the vulgar language, vulgar, dirty words constantly; Some college students pay great attention to personal hygiene, and the dormitory is dirty and disordered, but they don't take the initiative to clean up; Their own items are regarded as treasures, but it is no pity to damage the public property of dormitory, etc. These are easy to have a bad impact on the moral quality of the students in the dormitory, so it is easy to form a "poor nest" phenomenon (3) Dormitory has a profound influence on students' living condition and psychological quality. Half of college students spend half of their time in dormitories. Dormitory is not only a place for students to rest, but also a place for students to study, entertain, carry out the second classroom activities and other cultural life. The formation and development of students' ideology, behavior, learning atmosphere and interpersonal communication are inseparable from the specific environment of dormitory. College students are similar in age and close to their cultural quality, which is easy to produce psychological interaction. If a dormitory group is harmonious, the dormitory will become a "haven" for college students. They are willing to tell you their happiness and sorrow here, in order to get understanding and support from the collective. However, if they are not harmonious and ignore each other, and only regard the dormitory as a sleeping nest, students who live in such an environment for a long time will form a lonely and selfish personality.

Generally speaking, dormitory factors play the most important role in the formation and change of College Students' social network. On the one hand, for the class that has centralized accommodation at the beginning, the social network among students in the class is basically formed by the dormitory, and the dormitory is the main factor of social network convergence; On the other hand, for the class that has been used for decentralized accommodation at the beginning, there is no obvious clustering characteristic of the social network among the students. When the social network between the classes is adjusted to centralized accommodation in the later period, the social network between the class students gradually reflects the strong dormitory clustering characteristics. This shows that dormitory, as the most important place for college students' Extracurricular behavior, has not only limited its influence on the life level, but also permeates into the class, the most important basic organization of university learning. This discovery further integrates dormitory and class, dormitory and classroom, learning and life, education and teaching, management and service The boundary between ideological education and professional education provides empirical basis.

\section{Funding}

The Project Supported by Key Topics In The 13th Five Year Plan Of Jiangxi Education Science In 2019 (Grant no. 19ZD069) and The Key Topic Of Teaching Reform In Jiangxi Higher Education (Grant no. JXJG-19-17-2).

\section{References}

[1] Ministry of education, Ministry of education's notice on strengthening the management of College Students' accommodation (Jiao she Zheng [2004] No. 6), June 7, 2004.

[2] Ministry of education, general office of the Ministry of education on Further Strengthening the management of College Students' accommodation.

[3] You Fazhan, Wang Peng, Zhang Jie, Yan Yanglong, Yang Haojie. Research on the current situation and influencing factors of male dormitory relationship in Colleges and universities [J]. Modern business industry, 2018, 39 (26): 63-64.

[4] Ran Xiaoli. Analysis on the causes and Countermeasures of interpersonal relationship in college students' dormitory [J]. Contemporary education practice and teaching research, 2018 (06): 224-225.

[5] Yang Shaoqing, Zhang Jingxia. Relationship between dormitory and subjective well being of college students [J]. Chinese Journal of health psychology, 2012, 20 (12): 1905-1907.

[6] Zhang Dandan, Li Xiaoxi, Wei Shasha. Relationship between dormitory relationship and learning attitude and learning efficacy [J]. Chinese Journal of health psychology, 2012, 20 (10): 1585-1588.

[7] Pan Wei, Gao Xuemei. Interpersonal relationship, learning adaptation and academic achievement in college dormitory [J] Chinese Journal of health psychology, 2017, 25 (01): 73-76.

[8] Huang Yanfei, Zhang Tong. The practice and path of Ideological and political work entering students' dormitories in Colleges and Universities -- Taking the residential guidance work of Renmin University of China as an example [J]. Beijing Education (moral education), 2021 (04): 37-40.

[9] Lei ting. The construction and research of dormitory culture in Higher Vocational Colleges -- Based on the perspective of three complete education [J]. Chinese Journal of multimedia and network teaching (zhongxunjiao), 2021 (04): 205-207.

[10] Liu Juan. Correctly handling dormitory contradictions and guiding students to grow healthily: a case study of political counselors in Higher Vocational Colleges [J]. Engineering technology research, 2021, $6(05): 226-227+241$. 
[11] Jin Zhuan, Liu Changhua. Educational functions and methods of dormitory management in Colleges and Universities under the new situation -- Taking "dormitory revolution" of Anhui art college as an example [J]. Xueyuan, 2021, 14 (01): 67-69.

[12] Song Yuan. Exploration and practice of the guiding management mode of College Students' dormitory under the concept of establishing morality and cultivating talents -Taking Chengde Petroleum College as an example [J]. Scientific consulting (Science and technology · Management), 2020 (11): 52-53.
[13] Liu Qiming. Research on the construction of community management mode of student dormitory [J]. Zhili, 2020 (24): 86-88.

[14] Wang Shanshan, Li Fang. Analysis of interpersonal relationship in students' dormitories of Higher Vocational Colleges from the perspective of social psychology [J]. Style of science and technology, 2020 (18): 254-255.

[15] Huang Shaoxia. Feasibility analysis of intelligent management mode of college dormitory [J]. Science and technology information, 2020, 18 (18): $224+226$. 